\title{
Análisis del aprendizaje organizativo en el marco de la modernización de la gestión pública. El caso de la Administración Tributaria española
}

\section{José Prieto Jano * y Elena Revilla Gutiérrez **}

\section{Introducción: la necesidad de aprendizaje}

Cuanto más cerca estamos del final del siglo $\mathrm{xx}$, menos exagerado resulta decir que el ritmo y el alcance del cambio que está ocurriendo actualmente en las organizaciones y en el trabajo que ellas desarrollan no tiene precedentes históricos. Se trata de un cambio permanente, acelerado e interdependiente, que requiere que las organizaciones se adapten para sobrevivir. Puesto que la experiencia ha demostrado que las viejas soluciones simplemente no funcionan en la resolución de los nuevos problemas, y la única forma de conseguirlo es a través de un aprendizaje continuado, éste ya no puede ser una elección, sino una necesidad. Las organizaciones que quieran desarrollar su capacidad de aprendizaje deben ser capaces de cuestionar su pasado, innovar, tener una gran sensibilidad para inducir aspectos sobre las tendencias del entorno, estilo de vida, tecnología, hábitos de actuación, y una cierta voluntad de especular y moverse en lo desconocido -al menos en lo no habitual- y, por tanto, con un amplio margen de incertidumbre y ambigüedad.

De acuerdo con esto, resulta interesante observar cómo en los últimos tiempos se está generando una dinámica orientada a fomentar la capacidad de aprendizaje en la organización como uno de los elementos críticos para mantener a la organización en un estado de mejora continua. Las organizaciones que institucionalizan el aprendizaje y adoptan una actitud abierta al cambio se adaptan mejor al entorno. Obsérvese que esta necesidad de «aprendizaje continuo» implica que, en las organizaciones que emprenden este camino, el aprendizaje no tiene un punto final, no se formula para llegar a un destino concreto; el aprendizaje se institucionaliza.

Ninguna organización, con independencia de cuál sea su condición de pública o privada, puede estar al margen de esta necesidad de adaptación y de aprendizaje. El modo en que las organizaciones desarrollan su capacidad de aprendizaje varía de unas a otras, siendo muchos los factores que inciden en esta forma de hacer. Entre ellos, destaca la cultura, el proceso evolutivo; el entorno -interacción con el mercado, la pluralidad de agentes públicos/privados, participación social.

$\mathrm{Si}$ bien es cierto que, desde una perspectiva general, el tema del aprendizaje está de plena actualidad, poco ha sido dicho a este respecto de las organizaciones públicas. En este trabajo, nos proponemos estudiar la gestión del aprendizaje en la organización pública y, en particular, en la Administración Tributaria española. Así, seguidamente explicaremos cómo se encuentran configurados la Administración Tributaria y su entorno como parte de la Administración Pública española. A continuación, en el apartado ter- 
cero proponemos un modelo de aprendizaje que explica cómo las organizaciones, a partir de sus trabajadores directos, desarrollan su capacidad de aprendizaje. Una vez se tiene esto claro, es labor de la organización el diseño y desarrollo de unos procesos y estructuras que faciliten o permitan el aprendizaje. En este sentido, en el apartado cuarto, se analiza cómo estas estructuras pueden impulsar o, por el contrario, bloquear el proceso de aprendizaje en el marco de la Administración Tributaria. Finalmente, a modo de conclusión, terminamos proponiendo una serie de líneas de acción que guíen a los directores y gestores públicos para desarrollar la capacidad de aprendizaje y adaptación de la Administración Tributaria a estas nuevas premisas.

\section{La Administración Tributaria}

La Administración Tributaria actual, la Agencia Estatal de Administración Tributaria (AEAT), cuya creación tuvo lugar mediante el artículo 103 de la Ley 31/1990, de 27 de diciembre, de Presupuestos Generales del Estado para 1991. Es un Ente de Derecho público ${ }^{1}$, con personalidad jurídica propia y plena capacidad pública y privada. Se integra en las Administraciones Públicas centrales y se adscribe al Ministerio de Economía y Hacienda. Está encargado de la gestión y aplicación del sistema fiscal español. Su aparición en 1991 se enmarca dentro del proceso de modernización de la Administración Pública española planteado durante la década de los años noventa.

Entre los objetivos que tiene encomendados esta organización son de destacar los siguientes:

1. La aplicación efectiva del sistema tributario sobre todos los contribuyentes; objetivo dentro del cual se inserta la estrategia de lucha contra el fraude fiscal.

2. La disminución de los costes indirectos para los contribuyentes, derivados de las obligaciones tributarias para el fomento del cumplimiento voluntario de dichas obligaciones.

En este sentido, la Administración Tributaria, tal y como se encuentra configurada, se puede entender como una organización prestadora de servicios orientados a facilitar al contribuyente toda la información necesaria para el cumplimiento de las obligaciones tributarias, que sean de su competencia. Su eficiencia no depende únicamente del logro de los objetivos planteados en líneas precedentes, sino también del procedimiento aplicado en sus actuaciones o, lo que es lo mismo, de la sujeción a las normas jurídicas procedimentales ${ }^{2}$ y de la búsqueda del interés público.

Para la consecución de estos objetivos, la Administración Tributaria debe adaptar su esfera interna, condicionada por la asignación y gestión de sus recursos humanos y materiales, a su esfera externa, mediatizada, esta última, por el continuo flujo de intercambio de actuaciones entre Administración y contribuyentes. Dada la importancia de este proceso de adaptación Administración Tributaria-entorno, se procede a continuación a describir brevemente el contexto interno y externo en el que se mueve la Administración Tributaria española.

\subsection{La Administración Tributaria y su entorno}

En su esfera interna, la Administración Tributaria se caracteriza por una gran autonomía en aspectos tales como el régimen de personal y el de contratación, así como un sistema de financiación cuya novedad radica en que cuenta, entre otros recursos, con un porcentaje, estipulado anualmente por la Ley de Presupuestos Generales del Estado, de los recursos obtenidos por la recaudación ejecutiva de las deudas tributarias.

Con estas consideraciones, en el ámbito interno, la Administración Tributaria está compuesta de una organización central con funciones de coordinación y dirección, y en algunos casos de gestión directa del sistema tributario; y una organización periférica en la que se establecen tres niveles de administración con competencias propias en la gestión de sistema, que son: Delegaciones Especiales, Delegaciones y Administraciones.

Por su parte, el contexto externo en el que se mueve la Administración Tributaria está condicionado por los siguientes factores: la realidad sociopolítica del Estado y su estructura; el contexto económico de nuestro país; los productos resultantes de la interacción de las diversas políticas, principalmente, los derivados de las relaciones entre la Política Fiscal, la Política Monetaria, la Política Social y la Política Exterior; la regulación jurídica de las relaciones económicas y sociales; y, por último, la sociedad, desde el punto de vista de su percepción de la Administración Tributaria y del sistema fiscal, y de su percepción de las actuaciones emprendidas por ésta.

De los aspectos referenciados, y en lo que se refiere al cometido de este trabajo, cobra especial interés el entorno desde la óptica de la sociedad, de los ciudadanos, de los contribuyentes, a los que van destinadas las actuaciones tributarias en su diverso carácter; porque dicho entorno se muestra beligerante, en la medida en que las relaciones entre los contribuyentes y el Estado están sustentadas en el intercambio de impuestos-servicios.

El entorno social de la Administración Tributaria se define por las siguientes características:

- Es eminentemente dinámico, puesto que está sujeto a rápidas transformaciones, entre otras, de carácter económico, político y tecnológicas.

- Es complejo en la medida en que las relaciones Administración-contribuyente para su comprensión necesitan de una información cualificada, que haga comprensible dichas relaciones. 
- Presenta una diversidad de mercados, traducida en la amplia gama de clientes, contribuyentes, a los que se debe de controlar a través de la comprobación de los datos que éstos muestran en su autoliquidación impositiva, además de aquellos sujetos que ocultan su declaración impositiva, y sobre los que han de realizar tareas de investigación fiscal.

- Es bostil, puesto que los contribuyentes se muestran reticentes al pago de los impuestos exigidos para financiar los gastos y servicios públicos que el Estado ofrece. A esto se une la imposibilidad de predecir el comportamiento de los individuos ante las obligaciones fiscales influenciadas por factores económicos, sociales, psicológicos, políticos, etc.

El entorno social, así definido, ejerce su influencia sobre la Administración Tributaria a modo de control, a través de la conformación de la opinión que de ella tiene el colectivo de contribuyentes. De esta forma, el seguimiento y la evaluación del estado de percepción de los ciudadanos en su conjunto respecto a su Hacienda sirven a ésta para conocer la imagen de su gestión interna y la reacción de los individuos ante sus actuaciones. Los resultados de tal seguimiento implican que la Administración Tributaria deberá tomar, en su caso, las medidas correctoras oportunas; proponiendo cambios en la política fiscal, en las leyes fiscales y en las actuaciones administrativas (BARCELAY, 1991) al igual que lo hiciera un sistema de retroalimentación. Este proceso interactivo Administración-entorno social se constituye como un proceso de adaptación continuo en el que cada uno de los agentes - Administración y contribuyentes (sociedad) - incorpora en sus fueros internos, a través del aprendizaje, nuevas formas de hacer y de actuar.

Los programas de seguimiento emprendidos por la Administración Tributaria para conocer el estado de percepción de los ciudadanos respecto a su Hacienda son del siguiente tipo (GAITEIRO ForTES, 1990: 238):

1. Programas de investigación, que pretenden evaluar los estados de opinión de los ciudadanos acerca de la Administración Tributaria y su funcionamiento. Entre los programas de seguimiento y control de la opinión de los contribuyentes pueden citarse los siguientes (GATTEIRO FORTES, 1990: 239): sondeos de opinión sobre la Hacienda Pública; sondeos de opinión sobre los servicios de información y asistencia al contribuyente; investigaciones sobre la incidencia de las distintas campañas de información y seguimiento de las noticias tributarias publicadas en la prensa.

2. Programas de explotación de datos recogidos en los Servicios de Información y Asistencia al Contribuyente. En el ámbito de la gestión tributaria, el Servicio de Información Tributaria consiste en resolver las dudas y cuestiones que los contribuyentes plantean, al personal de la Administración Tributaria, a la hora de cumplir con las obligaciones fiscales impuestas. El Servicio de Asistencia Tributaria, por su parte, consiste en poner a dis- posición del contribuyente una serie de servicios para facilitar el cumplimiento voluntario de las obligaciones tributarias.

Las tareas de información y asistencia al contribuyente suponen un beneficio para la gestión tributaria, entendida ésta en sentido amplio. Permiten la normalización de los procedimientos gestores, lo que redunda en una mayor eficiencia y eficacia del personal, a la vez que introducen cambios en los métodos de control de rentas desde el Departamento de Gestión Tributaria, garantizando el máximo aprovechamiento de la información incluida en las bases de datos y proporcionadas por los propios contribuyentes. De cara al exterior, el Servicio de Información y Asistencia al Contribuyente ayuda al correcto conocimiento de la normativa fiscal en la medida en que contribuye a eliminar los errores de los contribuyentes y permite disminuir el número de actuaciones posteriores de comprobación.

3. Programas de análisis del comportamiento fiscal de los contribuyentes que se efectúa mediante la explotación informática de los datos tributarios en poder de la Administración. La información facilitada por los contribuyentes en sus distintas autoliquidaciones tributarias permite conocer la evolución temporal del grado de cumplimiento de sus obligaciones tributarias. En consecuencia, esto permite analizar el impacto que han tenido las distintas actuaciones de la Administración Tributaria en su lucha contra el fraude fiscal, así como la mejora del comportamiento fiscal del contribuyente, y adoptar las medidas necesarias para mejorar el mismo.

Mediante la obtención de estadísticas sobre las autoliquidaciones de impuestos y, especialmente, de las bases imponibles declaradas por fuentes de renta, se puede realizar un análisis comparativo entre dos o más ejercicios, al objeto de observar la evolución del comportamiento fiscal de los contribuyentes. Además, de forma indirecta, los datos estadísticos permiten observar cuál ha sido el efecto de las campañas publicitarias efectuadas con carácter previo, durante los períodos de declaración.

Una vez determinados los medios con que cuenta la Administración Tributaria para sondear el entorno social y conocer la opinión de los contribuyentes acerca de sus actuaciones, el paso siguiente es determinar cómo está configurada dicha opinión, lo que, en definitiva, nos lleva a analizar la cultura fiscal de la sociedad española.

\subsubsection{La cultura fiscal y su relación con la Administración Tributaria}

El elemento básico de la cultura es el sistema de valores compartidos. La cultura es el modo en que la sociedad vive e incluye en su haber las relaciones entre valores, roles y normas de comportamiento. En el caso de la sociedad española, la cultura fiscal presente, en la actualidad, es el producto de diversas acciones emprendidas por la Hacienda Pública y percibidas por 
los ciudadanos a lo largo de, casi, dos décadas desde que se inició la reforma que ha generado el sistema fiscal vigente, y de la configuración de la actual Administración Tributaria encargada de aplicarlo ${ }^{3}$. Dicha configuración, a su vez, es el resultado de los cambios en la estructura de la organización y del sistema fiscal que paralelamente se fueron produciendo fruto de las transformaciones surgidas en la economía nacional e internacional, la política, la Administración Pública y en la conciencia social de la sociedad española.

La prueba de los cambios sufridos por la estructura de la Administración Tributaria, como consecuencia de su adaptación a las circunstancias cambiantes del entorno, se muestra en el análisis gráfico del proceso evolutivo de la organización en sucesivas etapas que van desde que se inició la Reforma Fiscal hasta la aparición de la Agencia (Figs. 1, 2, 3 y 4).

En la Figura 1, se parte de la consideración de un mundo con tres agentes, a saber: instituciones políticas, Administración Tributaria y sociedad civil, para describir el fenómeno de interacción entre la organización tributaria y su entorno. En este momento anterior a la reforma fiscal, las inconexiones entre los tres tipos de agentes tenían su fundamento, principalmente, en su disposición para la elaboración y aplicación de la normativa al uso. Asimismo, la Figura 1 muestra las relaciones entre los agentes mencionados. Merece destacar que estas relaciones se suceden en una única dirección que va desde las instituciones hasta la sociedad, pasando por la Administración.

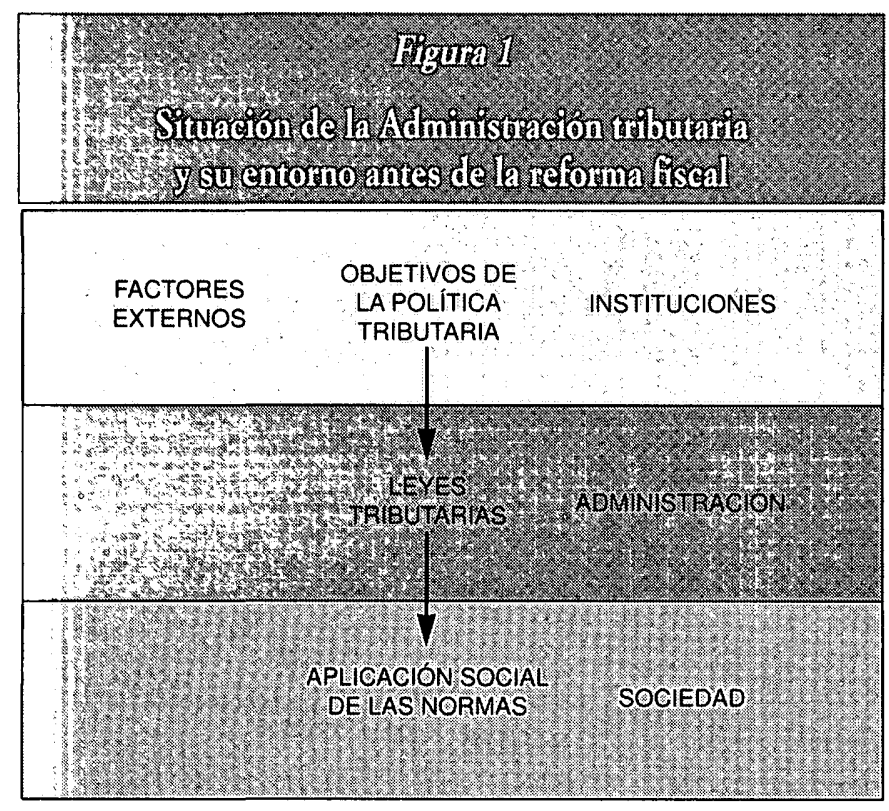

La Figura 2 muestra la etapa de la Reforma. En ella, se puede observar, a diferencia de la anterior, que se introduce un mecanismo de retroalimentación, mediante el cual la percepción social del tributo incide en los objetivos de la política fiscal, a través de las instituciones políticas. Ya, en este momento, el carácter democrático introducido mediante la creación de las recientes Cortes Generales permitía incluir como primeros cometidos la puesta en marcha de la Reforma Fiscal. La influencia de la percepción social llega también a la Administración Tributaria encargada de elaborar y aplicar las normas fiscales, de nuevo a la sociedad.

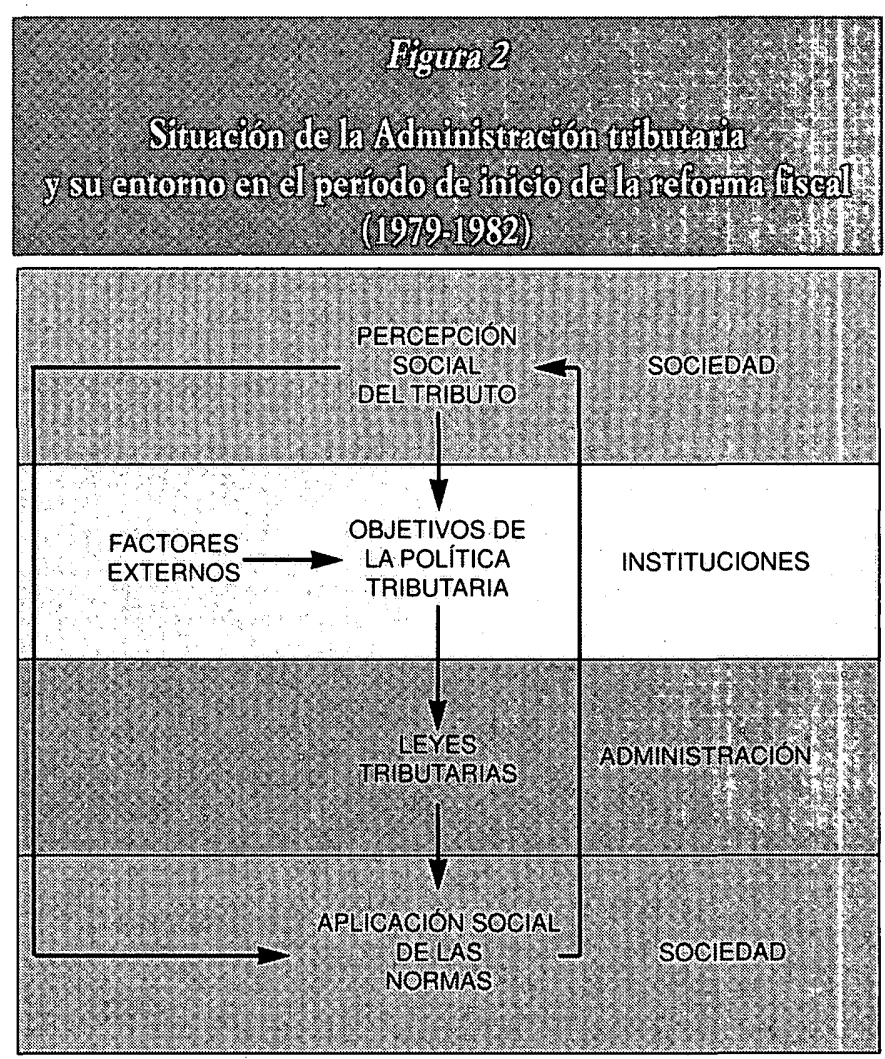

La Figura 3 muestra la introducción en los esquemas anteriores (Fig. 1 y Fig. 2) de una nueva estructura organizativa adaptada a las necesidades que fueron surgiendo a lo largo del período considerado (1982-1989), como garante de la aplicación de la normativa fiscal ${ }^{4} \mathrm{y}$ en cumplimiento del objetivo de lucha contra el fraude.

La Figura 4 muestra la inclusión de nuevas variables en los esquemas que se han venido exponiendo en líneas precedentes. Estas nuevas variables, cuales son las actitudes, valores y objetivos de los contribuyentes, además de la toma de decisiones de gestión por parte de la Administración Tributaria, aparecen en el nivel correspondiente al agente «Administración» y se derivan de la percepción que la sociedad tiene del tributo.

Analizada brevemente la evolución de la estructura de la Administración Tributaria, conviene volver a retomar el tema de la cultura fiscal. Entre los factores elementales, también conocidos como estructurales, que conforman el sustrato básico 


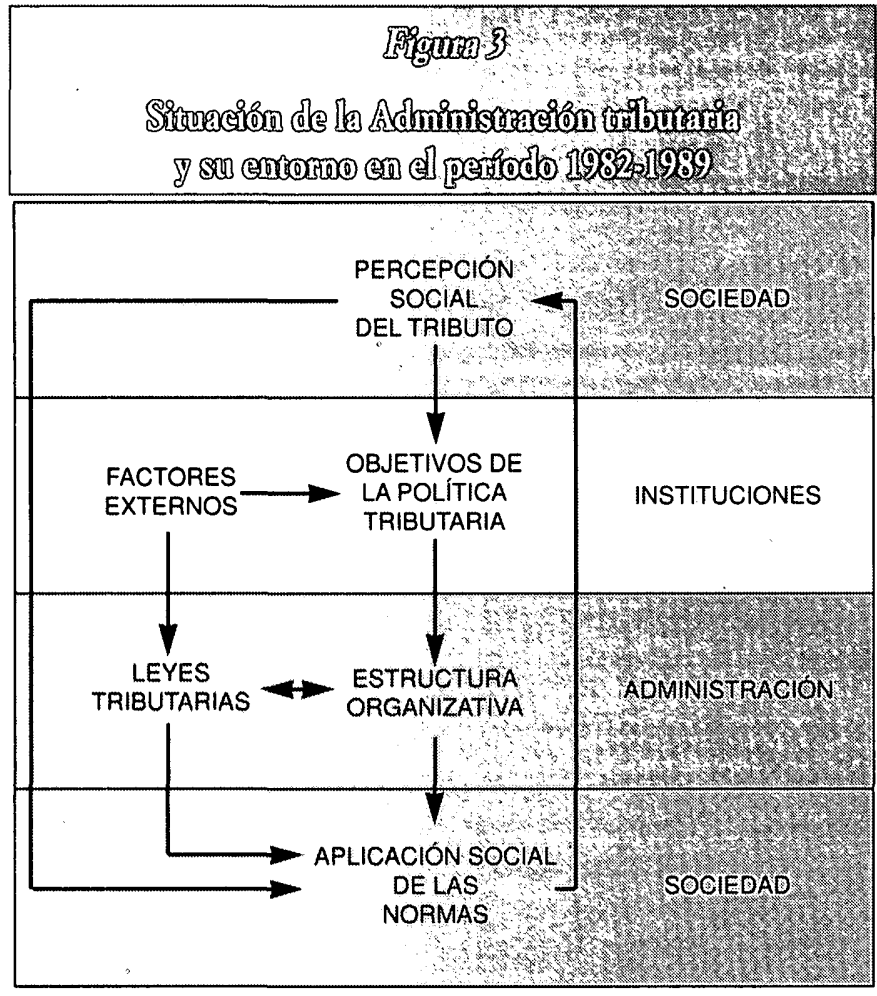

o estructura sobre la que se asienta la cultura fiscal, destacan, sobremanera, los siguientes:

- Las características básicas del sistema impositivo desde el punto de vista de la presión fiscal, justicia impositiva, simplificación tributaria, etc.

- Los servicios que el Estado ofrece en contrapartida a los impuestos pagados.

- La legitimidad con que cuenta el Estado entre los ciudadanos.

Aparte, se debe señalar que las relaciones ciudadano-Administración Tributaria están reguladas o intervenidas por otra serie de factores coyunturales, cuales son, entre otros:

- El desarrollo de las actuaciones inspectoras y judiciales en la persecución del fraude.

- El cuadro sancionador tributario aplicable a las infracciones tributarias cometidas.

- Los procesos de información y comunicación fiscales, que inciden de forma decisiva en la relación contribuyentes-Fisco.

- Desarrollo de los procedimientos de gestión y recaudación de los impuestos.

En la consecución de este proceso de adaptación en una Administración prestadora de servicios con su entorno, como es la Administración Tributaria, el proceso de aprendizaje cobra especial relevancia, puesto que éste va a ser fiel reflejo de la eficiencia de la organización en el proceso de resolución de los problemas; problemas, en gran parte, surgidos como con-

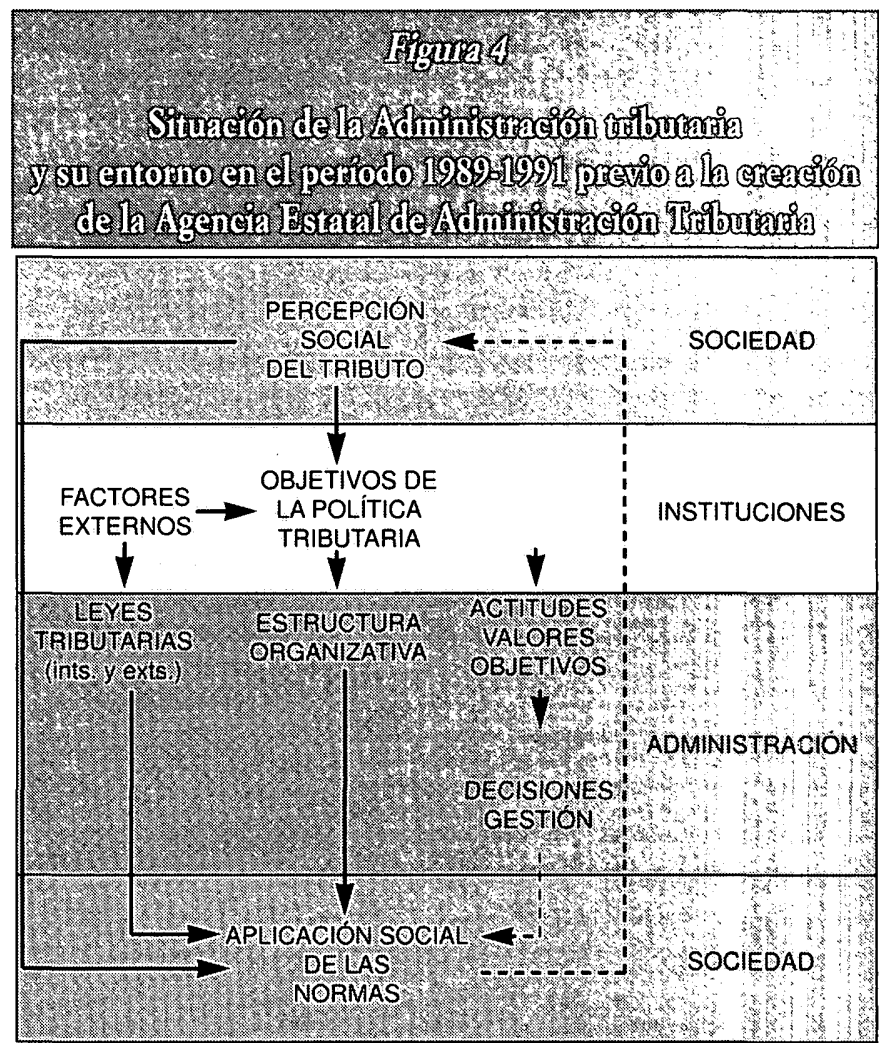

secuencia de la generalización del sistema de autoliquidaciones impositivas.

La importancia que el aprendizaje tiene en la labor de adaptación de la organización con su entorno nos lleva a querer entender este proceso con una mayor profundidad. En las líneas que siguen, se propone un modelo de aprendizaje que explica cómo las organizaciones aprenden a través de sus trabajadores directos.

\section{Premisas teóricas. Un modelo de aprendizaje organizativo $^{5}$}

Muchos autores, desde perspectivas diversas, han llegado a la conclusión de que el aprendizaje se logra fundamentalmente por medio de la resolución de problemas (JAIKUMAR y BoHN, 1986, y Hayes et al., 1988), explicando que éstos deben existir para que el aprendizaje ocurra. Siguiendo a PÉREz-López (1991), se define un problema como una situación que no es agradable para la persona. De acuerdo con esto, el proceso de resolución de problemas consiste en transformar una situa- 
ción desagradable en una que es agradable 0 , hacer desaparecer las diferencias percibidas entre lo que es y lo que debería ser. Las situaciones no agradables aparecen como consecuencia del cambio. En la literatura científica, el cambio se ha venido analizando desde hace mucho tiempo bajo el epígrafe de innovación (MuÑoz-SeCA y Rrverola, 1997). Consecuentemente, nuevas formas de actuación ante situaciones nuevas o antiguas son necesarias para que se generen los problemas cuya resolución origina nuevo conocimiento.

El aprendizaje, así generado, crea una nueva mentalidad en la persona, modifica su forma de entender las cosas y afrontar las dificultades y, consecuentemente, cambia sus reglas de actuación. En esta línea, se encuentran las aportaciones de ENGLISH y ENGLISH (WEICK, 1991), quienes señalan que la prueba de que el aprendizaje ocurre no es el cambio de respuesta derivado de una alteración en el estímulo, sino más bien un cambio de respuesta cuando el estímulo permanece sin variación. Esta alteración es consecuencia del cambio que se produce en el agente que responde.

Ahora bien, en todo este proceso debemos reconocer un mecanismo de realimentación que empieza en el modelo mental del individuo y termina con su reforzamiento. El modelo mental, fiel reflejo de la base de conocimientos del individuo, representa la imagen que éste tiene del mundo, e incluye tanto el entendimiento explícito como el implícito. Las ideas de mejora dependen directamente del volumen de conocimientos del individuo, de modo que podemos pensar que cuanto mayor sea su dotación inicial, mayor será la comprensión del funcionamiento y status del entorno y, en consecuencia, la introducción de nuevos planteamientos de actuación. Se puede concluir, por tanto, que la solución del problema no es el único resultado de todo este proceso de aprendizaje, sino también el desarrollo de nuevos conceptos, relaciones, reglas de decisión y operación que incrementan la capacidad de aprendizaje del individuo, proporcionando una toma de decisiones más efectiva.

El aprendizaje organizativo es un proceso distinto de la simple colección de experiencias individuales de aprendizaje (SHRIVASTAVA, 1983). Aunque los individuos son los agentes a través de los cuales la organización aprende, el aprendizaje individual debe ser comunicado, compartido públicamente e integrado en rutinas organizativas para ser considerado organizativo. En esta linea, KIM (1993) señala que el aprendizaje organizativo requiere un intercambio de modelos mentales entre sus miembros. Compartir los modelos mentales es el único medio que garantiza a la organización una completa utilización de sus experiencias pasadas, de los conocimientos de sus miembros individuales. Sin ello, resulta difícil que la organización modifique sus rutinas y reglas de decisión y actúe de forma más efectiva.

Para que el aprendizaje ocurra a un nivel organizativo, el aprendizaje individual debe ser difundido e implementado en el hacer diario de la organización. Debe hacerse externo a la persona, de modo que su objetivación, concreción y transferibilidad permita transformar las reglas de decisión que durante un tiempo han regido el comportamiento de la organización y realizar acciones más efectivas. Su cometido es desarrollar la capacidad de una organización para tomar decisiones efectivas.
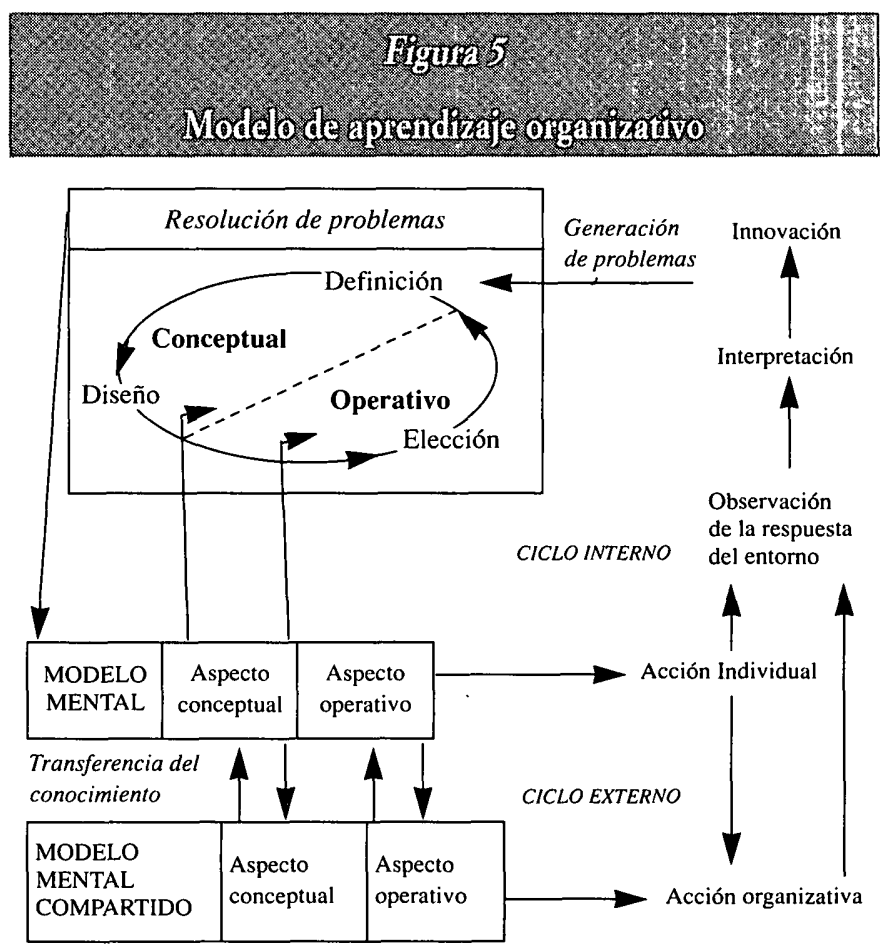

Este modelo de aprendizaje organizativo, presentado en la Figura 5, integra tres elementos claves: 1) la creación de problemas a través de la innovación y el pensamiento creativo, 2) la resolución de problemas, y 3) la transferencia de conocimiento desde el individuo a la organización.

La importancia de la generación de problemas deriva de la imperiosa necesidad de la organización de plantear nuevas formas de actuación que le permitan hacer frente a los cambios del entorno. Una vez asumida la necesidad que tiene la organización de seguir innovando, cobra importancia el proceso de resolución de problemas que genere los conocimientos requeridos por la organización para lograr un mayor grado de adaptación con su entorno. Por último, y al objeto de que el conocimiento generado con la resolución de problemas se traduzca en una mejor actuación de la organización, es primordial que el individuo transfiera su aprendizaje hacia la organización.

El aprendizaje organizativo resultante depende del funcionamiento de estos tres elementos considerados claves. Su fuerte interrelación requiere una gran coordinación de los mismos. Su funcionamiento espontáneo no garantiza el aprendizaje orga- 
nizativo. Fallos en la ejecución de este proceso o desviaciones en su dirección se traducen en pérdidas en la capacidad de aprendizaje de la organización y, en consecuencia, de adaptación a su entorno.

\section{Barreras al aprendizaje. El caso de la Administración Tributaria}

Una vez se tiene claro el proceso de aprendizaje por el cual una organización se alinea con su entorno, es competencia de la dirección el diseño de unas estructuras y procesos organizativos capaces de desarrollar eficientemente este proceso. Para seguir profundizando en este tema, a continuación trataremos de ver cómo este proceso se puede ver impulsado por la dirección $\mathrm{o}$, por el contrario, limitado por sus actuaciones. Para ello, en primer lugar, se señalaran cinco situaciones ${ }^{6}$ que, referidas a su lugar de actuación en este proceso, pueden bloquear la consecución de un efectivo aprendizaje: el aprendizaje bajo la incertidumbre, el aprendizaje bajo la ambigüedad, el aprendizaje situacional, el aprendizaje condicionado y el aprendizaje fragmentado. Seguidamente, y haciendo especial referencia a la Administración Tributaria, se identificarán los aspectos responsables de tal situación.

La relevancia de la aplicación de este análisis a la Administración Tributaria deriva de la necesidad de realizar un balance de los aspectos positivos y negativos y planteamientos de futuro en la gerencia de esta organización, que, por naturaleza y como se ha podido comprobar en el apartado segundo de este trabajo, es harto complicada en las relaciones, tanto «hacia fuera», Administración-entorno social, como «hacia dentro», referida a la asignación eficaz y eficiente de sus recursos materiales y humanos en las actuaciones que debe realizar.

Desde la creación de la Agencia Estatal de Administración Tributaria hasta la actualidad se puede decir que ha transcurrido un tiempo suficiente que nos permite valorar diversos aspectos de la gerencia desarrollada. Paralelamente, no se deben pasar por alto una serie de hechos acontecidos en la economía española que han incidido, tanto directa como indirectamente, en el quehacer de esta organización. El hecho más significativo, en cuanto a revolución de la gestión tributaria se refiere, lo encontramos en la incorporación de la economía española al Mercado Único europeo desde 1993. Resultados de esta incorporación ha sido la libertad de movimientos de capitales, la

desaparición de los controles aduaneros intracomunitarios, la armonización de los regímenes del IVA y de los Impuestos Especiales y la internacionalización de la información tributaria.

Asimismo, durante este período, la economía española ha sufrido una crisis económica agravada por la crisis social producida por los hechos escandalosos ocurridos en la política y en otras esferas de la sociedad, que no han pasado desapercibidos por la sociedad. La incidencia de la crisis económica en la gestión tributaria se manifiesta, en última instancia, en el agravamiento del déficit público, con la exigencia para la Administración Tributaria de garantizar la aplicación del sistema tributario con mínimos máximos de justicia.

\subsection{Barreras a la generación de problemas}

Con relación a la generación de problemas distinguimos dos situaciones capaces de bloquear el proceso de aprendizaje y las definimos como: el aprendizaje bajo la incertidumbre y el aprendizaje bajo la ambigüedad. En el primer caso, las cuestiones relativas a la falta de información impiden definir la reacción del entorno a la acción de la organización. En el segundo, temas relacionados con la complejidad de las interacciones de la organización con su entorno, dificultan su interpretación, dejando a ésta sin una base sólida sobre la que apoyar sus actuaciones.

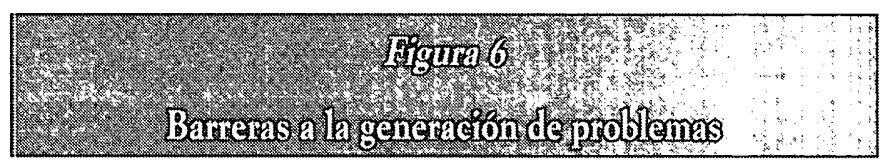

Aprendizaje en ambigüedad

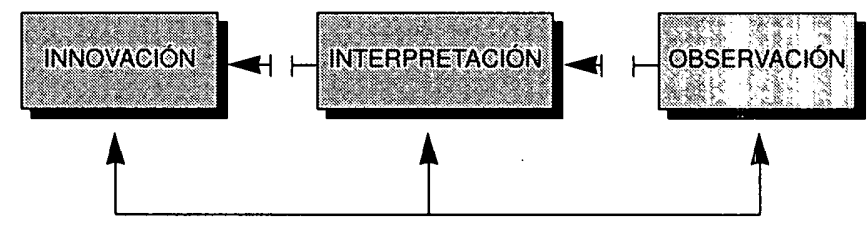

\subsubsection{Aprendizaje en incertidumbre}

Las organizaciones, de forma general, suelen presentar distintas actitudes en el acopio de datos del entorno. Mientras que unas muestran comportamientos pasivos, manteniendo unos sistemas de vigilancia rutinarios, otras adoptan una postura activa de búsqueda de información con sistemas de alerta no rutinarios en que apoyar su actuación (FAHEY y KING, 1977).

Cuando el entorno es dinámico, muchos de los aspectos que le definen varían y, con ello, también los modelos que explican su interacción con la organización. Esta sensibilidad 
de la organización a las variaciones del entorno exige una actualización constante de información que explique su nuevo ámbito de actuación al que adecuar su comportamiento. De acuerdo con el principio de «variedad relativa» de ASHBY, que afirma que la variedad del controlador debe ser del mismo orden que la variedad del controlado, cuanto más inestable sea el entorno mayores son los aspectos del entorno que deben controlarse y, por tanto, mayores serán las necesidades de información. En estas condiciones, una actuación pasiva de los miembros de la organización respecto a la recogida de datos del entorno puede suponer una falta de información relevante que impida la comprensión del funcionamiento del entorno y la adaptación de la acción de la organización a estas nuevas circunstancias. Para evitar que una carencia de datos pueda bloquear el desarrollo del conocimiento en la organización, ésta debe mantenerse activa en la recogida de información y establecer unos mecanismos de vigilancia que le permitan estar en continuo estado de alerta respecto a lo que allí ocurre (FAHEY et al., 1981).

Por contra, cuando la organización se mueve en ambientes definidos por unos modelos estables de comportamiento, la amenaza de no comprensión del entorno se ve difuminada. En estos casos de baja incertidumbre, donde la posibilidad de que ocurran sucesos inesperados es mínima, la existencia de actitudes menos activas en cuanto a la recogida de información no impide el planteamiento de ideas de mejora que generen problemas con cuya resolución la organización aprenda.

Centrándonos ya en el caso de la Administración Tributaria, su actuación en lucha contra el fraude, debe estar presidida por la necesidad de mantener un mínimo de justicia fiscal, observada ésta desde la garantía de los derechos de los contribuyentes (derecho a la intimidad, actuación objetiva y proporcionada de los órganos de la Administración, debido acceso a los Tribunales, etc.), hasta evitar que la presión fiscal con motivo de las rentas ocultas recaiga sobre determinado colectivo de contribuyentes, cuyas rentas es más difícil que escapen al control administrativo. Esta exigencia de atajar los fraudes calificados de mayor gravedad, los relacionados con los paraísos fiscales en operaciones financieras encubiertas y aquellos que estén insertos en actividades delictivas, requiere de una adecuada capacidad de investigación ayudada de una policía fiscal, tal y como se previó en la propia Ley de creación de la Agencia Estatal de Administración Tributaria.

La Agencia, desde el punto de vista organizativo, ha aprovechado la estructura de la antigua organización de la Hacienda Pública a nivel periférico mediante Delegaciones de la Agencia, Administraciones y Administraciones de Aduanas, permitiendo de esta forma una extensa implantación territorial. A pesar de la importante presencia de la Administración Tributaria, cabe destacar que no todas sus Administraciones disponen de todos los servicios con la preparación suficiente para resolver todas las tareas que se presenten, y el tamaño difiere de unas a otras, algunas de ellas necesitadas de la dotación de unidades operativas dado el gran volumen de trabajo que deben desarrollar.

Dada esta situación, y con el objeto de atajar el fraude fiscal, se necesitaría, en primer lugar, que en las Administraciones actuales haya una definición más precisa de los servicios que deben realizar y, en segundo lugar, que éstos se completen en su totalidad para cada una de éstas. Sin estos recursos, la Administración Tributaria difícilmente podrá disponer de la información que necesite para proponerse formas de actuación que garanticen una gestión de calidad, proporcionen a los ciudadanos la seguridad de que las normas fiscales se apliquen con objetividad y de forma efectiva, aseguren el cumplimiento de las obligaciones y sean implacables con los defraudadores. Cuando esto ocurre estamos ante una situación de un aprendizaje bajo la incertidumbre.

Asimismo, avanzar en el control de las operaciones internacionales e intracomunitarias, como consecuencia, estas últimas, de la apertura del mercado de capitales y la desaparición del control aduanero en las transacciones con mercancías, exige de la Administración Tributaria una especial formación en esta materia debido a su escasa experiencia en el tratamiento de la fiscalidad, tanto a nivel intracomunitario como internacional. Esta formación debe de ir dirigida tanto al personal inspector como a los jueces que han de imponer sentencias sobre delitos fiscales cometidos. Sin el conocimiento requerido para explorar las implicaciones de esta nueva realidad impositiva, no se podrá disponer de una amplia experiencia en la aplicación de las mismas y se generará un aprendizaje en incertidumbre. La aparición en la Administración Tributaria de nuevas formas de actuación derivadas de una aplicación generalizada de estas normas se ve bloqueada.

Si bien es cierta esta carencia en la formación a un nivel internacional, también es justo señalar que los recursos humanos de que dispone la Agencia presentan, por tradición, un alto grado de cualificación en cuanto a calidad, profesionalidad, dedicación en desempeño de las funciones encomendadas. Los recursos materiales de la Agencia, especialmente, los informáticos han alcanzado un alto nivel de desarrollo gracias a la incorporación de la alta tecnología a las tareas de gestión. Como resultado, la Administración Tributaria ha podido controlar un gran volumen de información, obtenida fundamentalmente de las autoliquidaciones de los impuestos realizadas por los contribuyentes, y situarse a la cabeza en cuanto a informatización se refiere respecto a los países de nuestro entorno.

\subsubsection{Aprendizaje en ambigüedad}

En los párrafos anteriores sólo hemos hecho mención expresa a una necesidad de información sobre la que apoyar la actua- 
ción de la organización cuando cambia su entorno. Esta recogida de información es suficiente cuando los vínculos que se establecen entre el entorno y la organización son bien entendidos, sin embargo, cuando las relaciones entre la organización y el entorno no están claras, cuando los sucesos que ocurren en el entorno no tienen una fácil explicación, además de un acopio de datos, se requiere un esfuerzo adicional de interpretación de los mismos. La imagen que la organización tiene de su entorno no explica bien su comportamiento, ni las expectativas creadas sobre él y, como consecuencia, exige cambios en las reglas de decisión. Al objeto de clarificar estas relaciones y evitar bloqueos en la generación de problemas, algunos autores opinan que la organización debe propiciar foros de discusión sobre los sucesos ${ }^{7}$, acordar una razonable interpretación y, en función de ello, plantear las acciones pertinentes (DAFT y LEN. GEL, 1986).

Sin duda, como ya se ha expuesto en el apartado de la Administración Tributaria, y sobre todo a partir de la creación de la Agencia, se ha avanzado mucho en el terreno del servicio al contribuyente. Prueba de ello es que la Administración Tributaria española, la Agencia, tiene asumidas la mayor parte de las tareas de gestión tributaria, en sentido amplio, de los tributos estatales, a diferencia de lo que ocurre en el resto de Administraciones Tributarias europeas que aparecen parceladas por tributos o por tareas o funciones. Esta ventaja es aprovechable principalmente por el colectivo de contribuyentes, que mantienen una relación con la Administración respecto a los tributos personales única y susceptible a mejoras en la aplicación del servicio, tal y como muestra la lucha contra el fraude y la mejor aceptación de impuestos por el contribuyente.

A pesar de estos avances, el servicio tributario ha de ir avanzando en el sentido de reducir la complejidad de las relaciones con los contribuyentes y encontrar planteamientos de actuación adecuados a las exigencias de cada ciudadano. Cada categoría de contribuyente debe recibir un tratamiento adecuado, tanto en las funciones de estímulo como a las dirigidas al control, que primariamente debe de ser preventivo. En la medida que se avance en este camino se impedirá que se produzca un apren. dizaje en ambigüedad.

\subsection{Barreras a la solución de problemas}

El aprendizaje condicionado y el aprendizaje situacional son las dos circunstancias identificadas como inhibidoras del aprendizaje que deberían desprenderse del proceso de resolución de problemas. El primero surge cuando se desvía la atención del nuevo problema hacia métodos ya conocidos de resolución, sin preocuparse de realizar una búsqueda activa de solución. En estos casos, las modificaciones que experimenta el modelo mental de las personas si no es nulo, es mínimo. El segundo, por su parte, aparece cuando se produce una falta de retención del aprendizaje generado con la resolución del problema en la memoria del individuo. Como consecuencia, no se produce ningún cambio en las reglas de decisión del individuo, limitándose la aplicación del conocimiento generado a una única situación concreta.

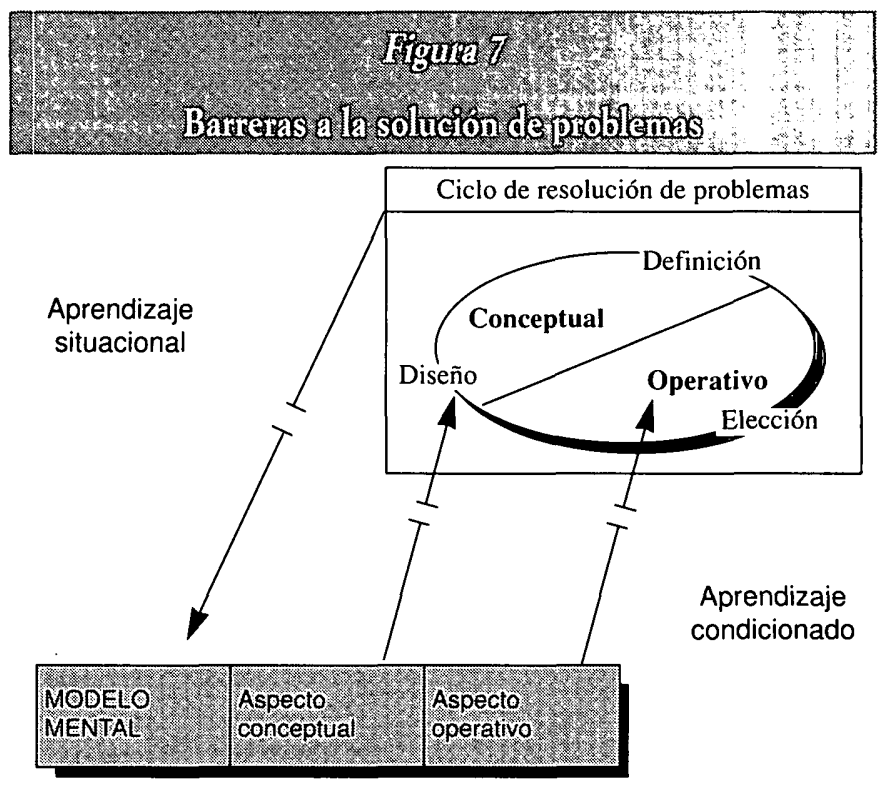

\subsubsection{Aprendizaje condicionado}

Cuando el entorno es dinámico, la aplicación de innovaciones - nuevas formas de actuación - no genera verdaderos problemas, sino sistemas de problemas, cuya solución exige su desagregación en un conjunto de problemas que se deben resolver por separado. De este modo, lo que empezó siendo un sistema de problemas es frecuente que, transcurrido el tiempo, degenere en un entramado de problemas cuya solución, aunque se realice a nivel individual, debe estar encaminada hacia la solución del sistema de problemas. En estas circunstancias, la cuestión que se nos plantea es resolver los problemas particulares no de forma aislada, sino formando parte de un complejo sistema de interrelaciones. Se requiere una solución de problemas integrada.

La necesidad de ligar los planteamientos de resolución de un problema determinado a los de los otros con él interconectados, obliga a la organización a ofrecer un posicionamiento a la persona encargada de resolver el problema a nivel individual, dentro de la arquitectura de todo el sistema de problemas. Una falta de comprensión del funcionamiento del sistema como un todo dificultará una resolución integrada del problema y, con cierta frecuencia, ofrecerá soluciones no válidas a nivel global. Es más, de no considerarse el problema inmerso en un sistema global como es la organización, es muy probable 
que en su resolución no se hayan tenido en cuenta todas las variables que le afectan y que sea difícil establecer un marco conceptual sólido donde apoyar la propia operativa de solución. En definitiva, se está bloqueando el proceso de aprendizaje.

A este respecto, resulta especialmente relevante estar atento a cómo las relaciones de autoridad de una organización y sus niveles de especialización pueden influir, tanto en la resolución de problemas como en su integración. Así, es relativamente común que las personas de mayor nivel jerárquico fijen el marco en el que se resuelven los problemas, imponiendo unos intereses sobre otros y condicionando el análisis y la interpretación de la información equívoca en torno a la resolución del problema. En estas condiciones, no es de extrañar que los individuos resuelvan los problemas sin una búsqueda activa de alternativas de solución, ignorando oportunidades de aprendizaje. La libertad de experimentar nuevos métodos y retar los paradigmas existentes es fundamental para que la resolución de problemas genere un nivel de aprendizaje alto.

Los obstáculos al aprendizaje que se derivan de la estructura jerárquica de la organización pueden amortiguarse con diseños organizativos más planos. En cuanto al derribo de las barreras procedentes de una excesiva especialización, IANsITI (1993) apunta que no se necesita tanto de especialistas en determinados campos de conocimiento, como de personas que posean un cierto grado de especialidad unido a una variada experiencia en otras disciplinas, directa o indirectamente relacionadas con su actividad principal. Para conseguir esto, la dirección tiene a su disposición herramientas de variada efectividad como pueden ser la formación de equipos multidisciplinares, el trazado de esquemas que recojan explícitamente las interrelaciones entre las distintas partes del sistema, la simultaneidad en la resolución de los problemas particulares, el establecimiento de responsabilidades y resultados comunes, etc.

Los sistemas de información desempeñan un papel relevante en la consecución de una resolución integrada de problemas. Si bien es cierto que una mayor calidad en el procesamiento de la información — cantidad, oportunidad, riqueza y bidireccionalidad- favorece una eficiente resolución de problemas, a veces, las organizaciones han mostrado una excesiva obsesión por el almacenamiento y distribución de la información, olvidándose de que el fin de la información es soportar su análisis y que las decisiones respecto a esa información dependen, en una gran medida, de la persona que lo realice (ZUBOFF, 1988). En este sentido, los sistemas de comunicación que se centran casi en su práctica totalidad en la distribución de información, olvidando que el conocimiento proviene del análisis y la interpretación de la información, es previsible que no propicien, al menos todo lo que sería deseable, entornos de debate que clarifiquen las situaciones confusas y ambiguas. Cuando esto ocurre se produce una inhibición del proceso de aprendizaje.
Teniendo en cuenta lo anteriormente expuesto y dado que la transformación de unos inputs de información en conocimiento es un proceso de construcción social que requiere diálogo, debate y discusión (Brown y DuguID, 1991), el corolario natural que se desprende es que la calidad del diálogo entre las personas que se ven afectadas por la resolución de un problema influye en la creación del conocimiento y en la bondad de la solución alcanzada para el mismo. El diálogo es una ayuda demostrada que evita la compartimentalización de los conocimientos en los especialistas e incorpora nuevas formas de pensar (ISAACS, 1993) y nuevas alternativas de acción.

En relación con la Administración Tributaria, uno de los aspectos negativos que se señalan como más incisivo en la marcha de la Administración Tributaria es el de una cultura compartimentada. La estructura de la Agencia es indefectiblemente un producto heredado de la antigua organización de la Hacienda Pública. Cada área funcional posee una cultura gerencial propia adquirida a través de la experiencia y formación distintas dependiendo del cuerpo o colectivo de funcionarios que la integran, y dirigida desde unos Centros con criterios y objetivos diferentes en todas ellas. Esta compartimentalización hace que los «árboles impidan ver el bosque», es decir, la falta de una visión global de la organización impide una coordinación en la resolución de problemas y como consecuencia se genere un aprendizaje condicionado.

Por está razón, la Administración Tributaria no debe malgastar sus fuerzas en el debate de la compartimentalización o divisionalización de las unidades, y acabar de una vez por todas con la definición del papel que debe tener cada una de las líneas directivas señaladas. Se deben crear líneas de coordinación e integración de los departamentos o servicios que permitan una visión global de la organización para ofrecer un «producto acabado» (DELGADo PACHECO, 1993), de forma que todas las actuaciones de gestión, inspección y recaudación estén dirigidas a un mismo objetivo, tanto a nivel estratégico como operativo para evitar la pérdida de control y de información que se produce en el paso de actuaciones un departamento a otro como consecuencia de la aplicación de los procedimientos de gestión, recaudación e inspección.

Desde esta perspectiva, no es difícil comprender que uno de los mayores problemas con que se encuentra la Administración Tributaria española, actualmente, se centra en el denominado alzamiento de bienes. Así, cuando mediante la aplicación del procedimiento de recaudación en vía ejecutiva se procede al embargo de los bienes, muchos de éstos, en ese momento, ya han desaparecido, deviniendo en incobrable la deuda pendiente. Asimismo, constituye otro problema de gran relevancia para la Administración Tributaria, el hecho de que la tarea de inspección tributaria se da por finalizada cuando ésta procede a levantar el acta correspondiente a la deuda objeto de investigación. A partir de ese momento, el Departamento de Inspección desconoce la 
suerte que ha corrido el acta de la deuda levantada que, muchas de las veces, resulta incobrable. Los problemas expuestos constituyen un claro ejemplo de aprendizaje condicionado.

\subsubsection{Aprendizaje situacional}

La realidad económica y social lleva cierta ventaja a la realidad jurídica debido a los trámites que esta última debe superar para poder regular los distintos hechos económicos y sociales que se van produciendo. Actualmente, el entorno con el que se enfrenta la Administración Tributaria es posible que sea más inestable y complejo e imprevisible de lo que ha sido hasta la fecha por la velocidad de los acontecimientos y la crisis en que está inmersa la economía. En este contexto, las conductas fraudulentas están en constante transformación muy por delante de la capacidad administrativa de control de éstas.

Esta excesiva velocidad en los acontecimientos ha podido obstaculizar el proceso de resolución de problemas y generar un aprendizaje situacional. PURSER (1991) señala que las presiones de tiempo a las que se ven sometidos muchos individuos en la organización les obliga a poner en marcha soluciones sobre las que muchas veces no han desarrollado un adecuado nivel de comprensión. La falta de un mínimo nivel de análisis en el que apoyar la solución adaptada puede provocar un cierta desconfianza en la solución adoptada e impedir su posterior aplicación para la resolución de otros problemas similares. Si bien es cierto que un determinado nivel de presión de tiempo puede acelerar el ritmo de resolución, ciertas investigaciones sugieren que esto es un factor motivador sólo hasta un determinado punto (KeLly y MCGRATH, 1985).

\subsection{Barreras a la transferencia del conocimiento}

Con relación a la transferencia del conocimiento, el aprendizaje fragmentado identifica situaciones en las que el aprendizaje derivado de la resolución de problemas está en posesión exclusiva de determinadas personas con las cuales la empresa establece una relación de dependencia que puede dar lugar a comportamientos oportunistas y a problemas de agencia.

Temas secretos, conflictos entre funciones y departamentos, falta de confianza en la información recibida o en las perspectivas recibidas tanto por canales formales o informales, desconfianza en los resultados de los individuos que llevan poco tiempo en la organización, rápidos cambios de puesto de trabajo -dentro o fuera de la organización- de las personas que poseen el conocimiento, falta de responsabilidad por los resultados, falta de cooperación, rechazo a cambios de comportamiento y un extremado stress tanto individual como organizativo son algunos de los motivos por los cuales la organización ve
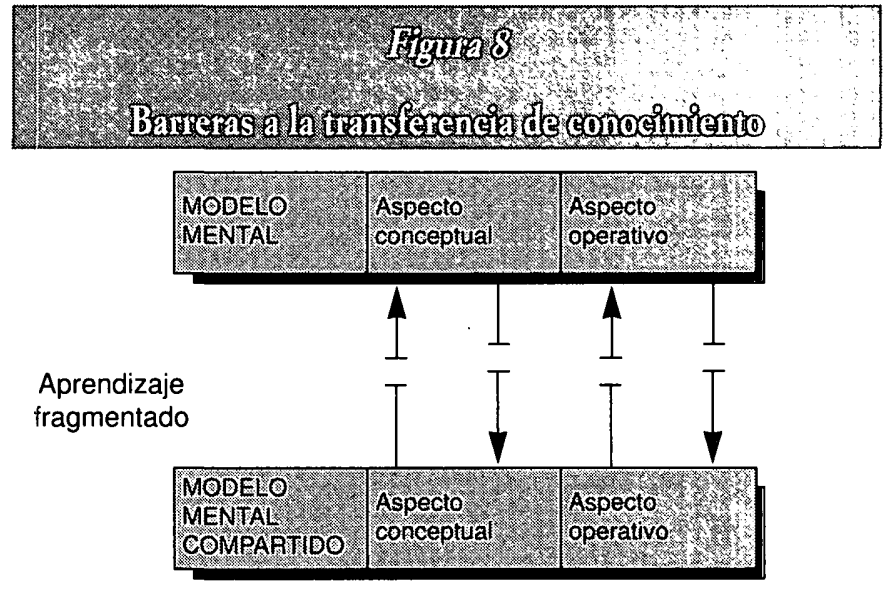

bloqueada la difusión del conocimiento de sus individuos (PuRSER et al., 1992). Estos hechos que, a simple vista, reflejan defectos en el sistema de planificación o en la estructura organizativa, responden, sólo a veces, a una incapacidad para relacionar las distintas partes de la organización. En otras ocasiones pueden surgir, bien por efectos indirectos de estrategias -intencionadas o no- por parte de los directivos a forzar rápidas soluciones a temas complejos, o bien por la creencia de que los especialistas en determinadas materias de conocimiento son los responsables directos de su aplicación.

En relación con este tema se puede decir que en la Administración Tributaria a nivel periférico se produce, en muchos casos, una duplicidad de autoridades; las autoridades territoriales, representadas por los Delegados Especiales, Delegados y Administradores, con frecuencia, entran en conflicto con las autoridades funcionales (Gestión, Recaudación e Inspección). Esto es debido a la reticencia que existe, por parte de quienes dirigen la Agencia, a romper con una estructura heredada del pasado. A pesar de que se repite una y otra vez que la modernización de la Administración debe empezar por el cambio de mentalidad de los directivos que la presiden, aún siguen produciéndose este tipo de ambigüedades en el ámbito gerencial y directivo. En estas circunstancias, se produce un aprendizaje fragmentado que impide el cambio de mentalidad del personal directivo.

\section{Conclusiones}

En este trabajo hemos estudiado la gestión del aprendizaje en la Administración Tributaria. Tras presentar un esquema general que explica cómo las organizaciones desarrollan su capacidad de aprendizaje para tomar acciones más efectivas y adaptarse mejor al entorno, se identifican una serie de situaciones que inhiben el aprendizaje y la comunicación, evitando un aná- 
lisis profundo de la información, la creatividad y el cambio necesario para desarrollarla con éxito. Su análisis en el caso de la Administración Tributaria nos ha llevado a proponer una serie de líneas de acción que guíen a los gestores públicos en su intento de mejorar la capacidad de aprendizaje y adaptación de la Administración Tributaria.

La existencia de una cultura gerencial propia en cada una de las áreas funcionales de la Agencia - gestión, inspección y recaudación - impide la realización de una adecuada coordinación entre áreas y la creación de una visión compartida en el seno de la Administración Tributaria española: Su solución requiere la creación de nuevas líneas de coordinación e integración de los departamentos y servicios que, permitan aprovechar los conocimientos adquiridos y la información disponible en cada una de las áreas funcionales y, a su vez, permitan la consecución de los objetivos propuestos para la organización tributaria con una mayor eficacia y eficiencia.
Asimismo, aun siendo conscientes de los importantes logros conseguidos en los últimos tiempos por el Servicio de Información y Asistencia al contribuyente que ofrece la Administración Tributaria española, su avance debe ir dirigido a reducir la complejidad de la normativa fiscal y de las relaciones con los contribuyentes; proporcionar a éstos un mínimo de justicia fiscal -atajando los fraudes fiscales calificados de mayor gravedad-; y garantizar los derechos de los contribuyentes ante los propios órganos de la Administración Tributaria.

Por último, se propone un mayor esfuerzo en la definición de las funciones las Administraciones de la Agencia que, evite solapamientos de servicios y duplicidad de autoridades con otras áreas de la organización tributaria. Su consecución exige que éstas cuenten con el mayor número de servicios, sobre todo, de información y asistencia a los contribuyentes y una preparación adecuada del personal para resolver los problemas y tareas que se presenten.
* Facultad de CCEE. Departamento de Economía Aplicada. Universidad de Valladolid.

*" Instituto de Empresa. Universidad de Valladolid.

1 La AEAT se constituye como un Ente público de los contemplados en el artículo 6.5 de la Ley General Presupuestaria. Esta norma recoge el régimen jurídico básico de los denominados «Otros Entes del Sector Público Estatal». Se trata de sujetos de Derecho que no son ni Organismos Autónomos ni Sociedades Estatales. Tienen personalidad jurídica propia y en todo lo que no regule específicamente su norma de creación se les ha de aplicar lo establecido en la Ley general Presupuestaria para la Sociedades Estatales.

${ }^{2}$ Las normas jurídicas procedimentales a las que se hace referencia en las líneas anteriores son las siguientes:

- La Administración Tributaria tiene asignadas las funciones de gestión, inspección, recaudación y para su regulación se rige por lo dispuesto en la Ley General Tributaria, en la Ley de Régimen Jurídico de las Administraciones Públicas y del Procedimiento Administrativo Común y en las normas dictadas en su desarrollo y ejecución.

- La contratación de la Agencia se rige por las normas de Derecho privado, ajustándose a los principios de publicidad, concurrencia y salvaguardia del interés público y para la homogeneización de comportamientos en el Sector Público.
- El patrimonio propio de la Agencia es gestionado por ella misma con arreglo a lo estipulado en la Ley de Entidades Estatales Autónomas.

- La Agencia puede constituir o participar en el capital de entidades que adopten la forma de sociedad mercantil y cuyo objeto social esté vinculado con los fines y objetivos de aquélla.

${ }^{3}$ Para una mayor información acerca del proceso evolutivo de la Administración Tributaria española veáse Prieto (1997), «Modernización y eficiencia de la Administración Pública. Especial referencia a la Administración Tributaria», Tesis Doctoral, Universidad de Valladolid.

${ }^{4}$ Dicha normativa en su mayor parte procedía de la etapa anterior, aunque se sigue avanzando en el producción normativa en este período.

${ }^{5}$ Para un mayor detalle de este modelo de aprendizaje organizativo, véase REVILLA (1996), Factores Determinantes del Aprendizaje Organizativo. Un modelo de Desarrollo de Productos, Madrid: Club Gestión de Calidad.

${ }^{6}$ Autores como MARCH y OLSEN (1975) y KIM (1993) señalan situaciones de bloqueo del aprendizaje organizativo similares.

${ }^{7}$ En el caso de la Administración Tributaria estos foros pueden estar propiciados desde dentro de la propia Administración, desde el ámbito académico y desde el ámbito profesional.

\section{Bibliografia}

AEAT (1992), Balance del primer año de gestión, Madrid: AEAT, Ministerio de Economía y Hacienda.

Barzelay, M. (1991), «La experiencia de modernización administrativa en el Departamento de impuestos de Minnesota», en La gestión tributaria y el servicio al contribuyente, Monografía No. 96, Madrid: IEF: 61-81.

Brown, J., y Duguid, P. (1991), «Organizational Learning and Communities of Practice: Toward a Unified View of Working, Learning and Innovation», Organization Science, 2, 1: 40-57
Daft, R. y Lengel, R. (1986), «Organizational Information Requirements, Media Richness and Structural Design», Management Science, 32, 5: 554-571.

De Juan Chocano, A. et al. (1994), «Cumplimiento fiscal voluntario de los contribuyentes españoles», Hacienda Pública Española, No. 131, IEF: 63-77.

Delgado Pacheco, A. (1993), «El futuro de la Administración Tributaria del Estado en el marco de la nueva Agencia», Punto de Encuentro, No. 2, Colegio de Huérfanos de Hacienda. 
Díaz YuBero, F. (1995), «Intervención de las Oficinas gestoras en la aplicación del sistema tributario estatal», en Cuadernos de Formación de la Inspección de Tributos, No. 32, Tema central, IEF.

FAHEY, L. W., y KING, W. R. (1977), «Environmental Planning for Corporate Planning», Business Horizons, 4, 20: 61-71.

GaITEIro ForTES, J. (1990), «Otros procesos de revisión gerencial», en Medición de la eficiencia y efectividad de la Administración Tributaria, XXIV Asamblea General del CIAT, Madrid: IEF: 227-245.

- (1991), «La modernización de la Administración tributaria española», en Jornadas para la modernización de las Administraciones Públicas, celebradas el 9, 10 y 11 de julio de 1990 en Cuenca, Madrid: MAP.

- (1992), «La Administración Tributaria en el marco de la organización de la Administración Pública», Cuadernos de Formación/Inspección de Tributos, No. 27, Madrid: IEF.

Hayes, R.; Wheelwright, S., y Clark, K. B. (1988), Dynamic Manufacturing: Creating the Learning Organization, New York: The Free Press.

Inssin, M. (1993), «Real-World R\&D: Jumping the Product Generation Gap», Harvard Business Review, mayo-junio: 138-147.

ISMACs, W. N. (1993), «Dialogue, Collective Thinking, and Organizational Learning», Organizational Dynamics, Autumn: 24-39.

JAikUmar, R., y BoHN, R. (1986), «The Development of Intelligent Systems for Industrial Use: A Conceptual Framework», Research on Tecbnological Innovation, Management and Policy, 3: 169-211.

Kelly, J., y McGrath, J. (1985), «Effects of Time Limits and Task Types on Task Performance and Interaction of Four Person Groups», Journal of Personality Socilogical Psycology, 49: 395-407.

KIM, D. H. (1989), «Learning Laboratories: Designing Reflective Learning Environments», en Miluing, P., y ZAHN, E. (eds.), Computer-Based Management of
Complex Systems: Proceedings of the 1989 International Conference of System Dynamics Society, Berlin: Springer-Verlag.

Muñoz-SeCA, B., y Rrverola, J. (1997), Gestión del Conocimiento, Biblioteca IESE gestión de empresas, IESE, Navarra University, Spain.

Lasheras Merino, Miguel A. (1991), «El cumplimiento voluntario de las obligaciones fiscales», en La gestión tributaria y el servicio al contribuyente, Monografía del IEF, No. 96: 91-100.

Pérez Menayo, V., y Bartolomé Llorente, J. (1996), «La gestión de la calidad total y su aplicación a la Administración Pública», en Lecturas de gerencia desde la Administración financiera, Madrid: IEF, Ministerio de Economía y Hacienda.

Pérez-López, J. (1991), Teoría de la Acción Humana en las Organizaciones: La Acción Personal, Madrid: Rialp.

Prieto Jano, M. J. (1997), «Modernización y Eficiencia de la Administración Pública. Especial referencia a la Administración Tributaria española», Tesis Doctoral. Universidad de Valladolid.

PurSER, R. (1991), «Redesigning the Knowledge-Based Product Development Organization: a Case Study of Sociotechnical Systems Change», Technovation, 11, 7: 403-415.

Purser, R., y PASMORE, W. (1992), «Organizing for Learning», en R. WOOdman y W. PASMORE (eds.), Research in Organizational Change and Development, vol. 6, Greenwich: JAI Press: 37-114.

ReVLlla (1996), Factores Determinantes del Aprendizaje Organizativo. Un modelo de Desarrollo de Productos, Madrid: Club Gestión de Calidad.

SHRIVASTAVA, P. (1983), «A Typology or Organizational Learning Systems», Journal of Management Studies, 20, 1: 7-24.

WEICK, K. (1979), The Social Psicology of Organizing, Addison-Wesley, Mass.

ZuBoff, S. (1988), In the Age of Smart Machine, New York: Basic Books. 
